\title{
COST Action TD1105: European Network on New Sensing Technologies for Air-Pollution Control and Environmental Sustainability - EuNetAir
}

\author{
Michele Penza ${ }^{1}$ - on behalf of EuNetAir Consortium \\ ${ }^{1}$ ENEA - Italian National Agency for New Technologies, Energy and Sustainable Economic \\ Development, Laboratory of Functional Materials and Technologies for Sustainable Applications, \\ Brindisi Research Center, PO Box 51 Br-4, I-72100 Brindisi, Italy \\ michele.penza@enea.it
}

\begin{abstract}
This is a short overview of the COST Action TD1105 EuNetAir - European Network on New Sensing Technologies for Air-Pollution Control and Environmental Sustainability - funded in the framework European Cooperation in the field of Scientific and Technical Research (COST) during the period 2012-2016. The international networking has involved more than 120 teams from academia, research, agencies and industry from 31 COST Countries and 7 Non-COST Countries (June 2015).
\end{abstract}

Key words: Sensing technologies, air pollution modelling, methods and protocols, air quality control, environmental sustainability

\section{Introduction}

The main objective of the Concerted Action EuNetAir is to develop new sensing technologies for Air Quality Control at integrated and multidisciplinary scale by coordinated research on nanomaterials, sensorsystems, air-quality modelling and standardised methods for supporting environmental sustainability with a special focus on Small and Medium Enterprises.

This international Networking, coordinated by ENEA (Italy), includes over 120 big institutions/organizations and over 200 international experts from 31 COST Countries (EU-zone: Austria, Belgium, Bulgaria, Croatia, Czech Republic, Denmark, Estonia, Finland, France, Germany, Greece, Hungary, Iceland, Ireland, Israel, Italy, Latvia, Luxembourg, The Former Yugoslav Republic of Macedonia, Netherlands, Norway, Poland, Portugal, Romania, Serbia, Slovenia, Spain, Sweden, Switzerland, Turkey, United Kingdom) and 7 Non-COST Countries (extra-Europe: Australia, Canada, China, Morocco, Russia, Ukraine, USA) to create a S\&T critical mass in the environmental issues.

This COST Action [1-4] (see logo in Fig. 1) will focus on a new detection paradigm based on sensing technologies at low cost for Air Quality Control (AQC) and set up an interdisciplinary top-level coordinated network to define innovative approaches in sensor nanomaterials, gas sensors, devices, wireless sensor-systems, distributed computing, methods, models, standards and protocols for environmental sustainability within the European Research Area (ERA). The state-of-the-art showed that research on innovative sensing technologies for AQC based on advanced chemical sensors and sensor-systems at low-cost, including functional materials and nanotechnologies for ecosustainability applications, the outdoor/indoor environment control, olfactometry, air-quality modelling, chemical weather forecasting, and related standardisation methods is performed already at the international level, but still needs serious efforts for coordination to boost new sensing paradigms for research and innovation.
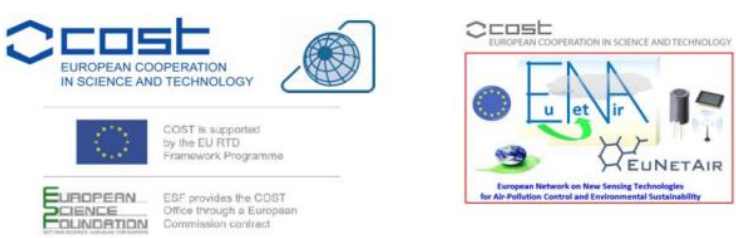

Fig. 1. COST Association, ESSEM Domain and Action TD1105 EuNetAir logo.

Only a close multidisciplinary cooperation will ensure cleaner air in Europe and reduced negative effects on human health for future generations in smart cities, efficient management of green buildings at low $\mathrm{CO}_{2}$ emissions, and sustainable economic development. 
The aim of the Action is to create a cooperative network to explore new sensing technologies for low-cost air-pollution control through field studies and laboratory experiments to transfer the results into preventive real-time control practices and global sustainability for monitoring climate changes and outdoor/indoor energy efficiency. Establishment of such a European network, involving Non-COST keyexperts, will enable EU to develop world capabilities in urban sensor technology based on cost-effective nanomaterials and contribute to form a critical mass of researchers suitable for cooperation in science and technology, including training and education, to coordinate outstanding $R \& D$ and promote innovation towards industry, and support policy-makers.

\section{Objectives}

The main objectives of Action include:

- to establish a top-level Pan-European multidisciplinary $R \& D$ platform on new sensing paradigm for AQC contributing to sustainable development, green-economy and social welfare

- to create collaborative research teams in the ERA on the new sensing technologies for $A Q C$ in an integrated approach to avoid fragmentation of the research efforts

- to train Early Stage Researchers (ESR) and new young scientists in the field for supporting competitiveness of European industry by qualified human potential

- to promote gender balance and involvement of ESR in AQC

- to disseminate $R \& D$ results on $A Q C$ towards industry community and policy makers as well as general public and high schools

\section{Working Groups}

The Action Workplan is organized in four complementary Working Groups (WGs), each devoted to a progressive development of synthesis, characterization, fabrication, integration, prototyping, proof-of-concepts, modeling, measurements, methods, standards, tests and application aspects. The four WGs with the specific objectives are:

- WG1: Sensor materials and nanotechnology

- WG2: Sensors, devices and sensorsystems for $A Q C$

- WG3: Environmental measurements and air-pollution modeling
- WG4: Protocols and standardisation methods

This Action focusses on the study of sensor nanomaterials and nanotechnologies exhibiting unique properties in terms of chemical and thermal stability, high sensitivity, selectivity. Nanosize effects of functional materials are explored for integration in the gas sensors at low power-consumption. Furthermore, specific nanostructures with tailored sensing properties will be developed for gas sensors and sensorsystems with advanced functionalities. Selected high-quality research products and innovative technologies developed by the partnership of COST Action TD1105 are shown in Fig. 2 and Fig. 3.

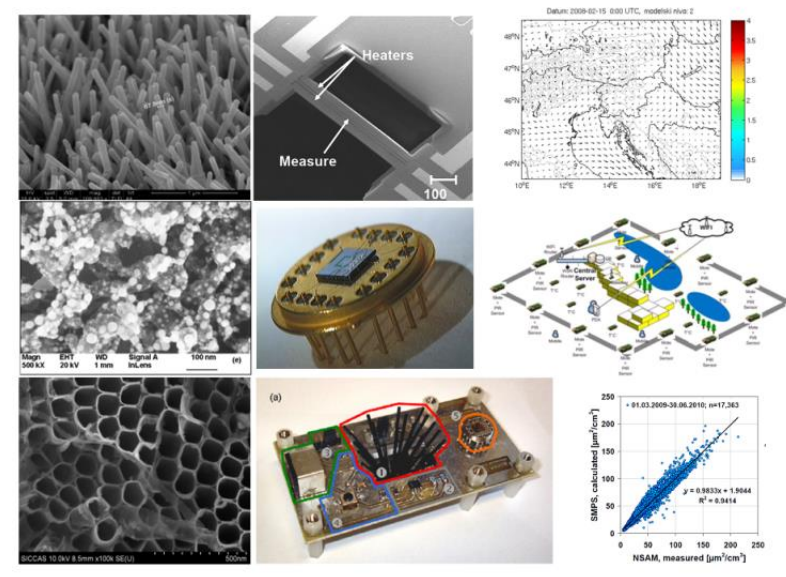

Fig. 2. Selected R\&D technological products developed by some partners (academia, research institutes, agencies, industry) involved in the COST Action TD1105 EuNetAir. Courtesy from EuNetAir partnership.

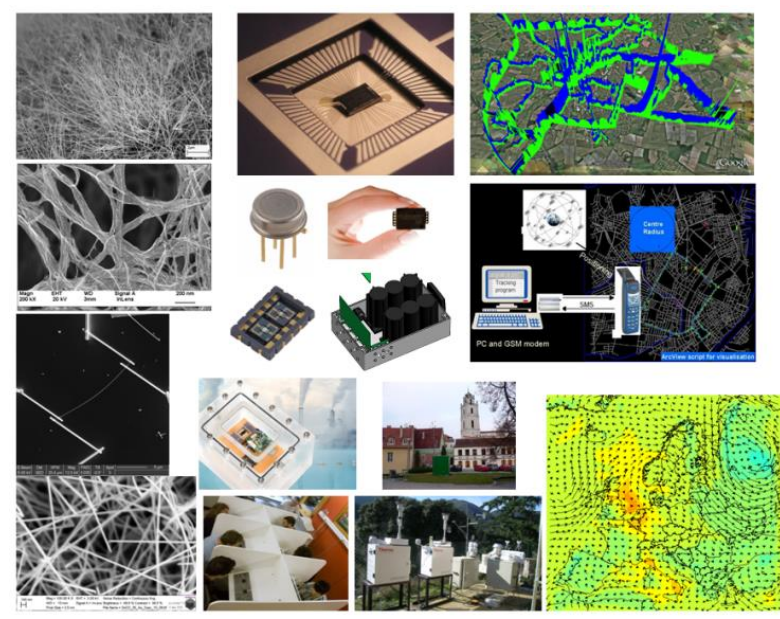

Fig. 3. Selected R\&D technological products developed by some partners (academia, research institutes, agencies, industry) involved in the COST Action TD1105 EuNetAir. Courtesy from EuNetAir partnership. 


\section{Special Interest Groups}

The COST Action TD1105 is composed by four multidisciplinary and focused Special Interest Groups (SIGs), each devoted to a thematic hottopic in the field of air quality control and related low-cost sensing technologies applications. The four SIGs with the specific objectives are:

- SIG1: Network of spin-offs

- SIG2: Smart sensors for urban air monitoring in cities

- SIG3: Guidelines for best coupling airpollutant and transducer

- $\quad$ SIG4: Experts comments for the revision of the air quality EU directive

The SIGs are intra-WGs, as shown in Fig. 4, with interdisciplinary themes of environmental interest including technologies, modeling, methods, standards and regulation.

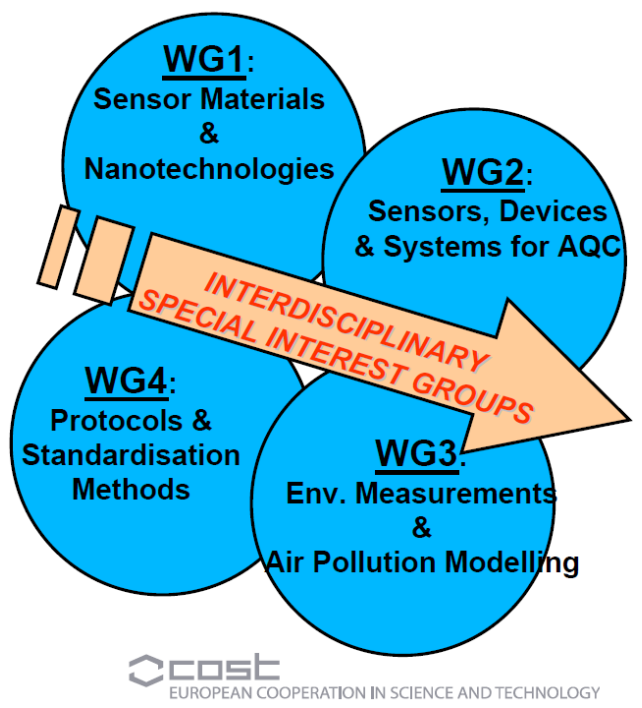

Fig. 4. WGs and SIGs structure of the COST Action TD1105 EuNetAir.

\section{Governance of COST Action TD1105}

The Action is governed by a Management Committee, led by the Chair and Vice-Chair, based on 58 Members and 33 Substitutes, nominated by COST National Coordinators (CNC) from 31 COST Countries. The Grant Holder $(\mathrm{GH})$ as Eurice GmbH (Germany) has been appointed for funding administration. A Core-Group has been established based on Chair, Vice-Chair, GH Representative, 4 WGs Leaders, STSMs Coordinator, Scientific Secretary, Webmaster. Moreover, a Steering Committee and various Ad-Hoc Groups are installed as follows as:

- Editorial Board

- Dissemination
- Training Schools

- Gender Balance

- Early Stage Researchers (ESR)

- $\quad$ Short-Term Scientific Mission (STSM)

- Intellectual Property Rights (IPR)

- $\quad$ Local Organizing Committee (LOC)

\section{EuNetAir Partnership}

At the date on June 2015, 123 big Institutions from 31 European Countries (Austria, Belgium, Bulgaria, Croatia, Czech Republic, Denmark, Estonia, Finland, France, Germany, Greece, Hungary, Iceland, Ireland, Israel, Italy, Latvia, Luxembourg, The Former Yugoslav Republic of Macedonia, Netherlands, Norway, Poland, Portugal, Romania, Serbia, Slovenia, Spain, Sweden, Switzerland, Turkey, United Kingdom) signed the Memorandum of Understanding (MoU). The Action participants are from 55 Universities (44\%), 39 Research Centers (32\%), 4 Environmental Agencies (3\%) and 25 SMEs (21\%) including 9 spin-offs. Additional 8 top-level Institutions from 7 Non-COST (Near Neighbour Countries and International Partner Countries) Countries (Australia, Canada, China, Morocco, Ukraine, Russia, USA) were involved: CSIRO, Chinese Academy of Sciences, National Research Center Kurchatov-Institute, O.M. Marzeiev Institute for Hygiene and Medical Ecology of Academy of Sciences of Ukraine, University of Agadir IBN Zohr, University of Waterloo, Southern Illinois University Carbondale, NASA Ames Nano Research Center. A panorama of the Action partnership is shown in Fig. 5.

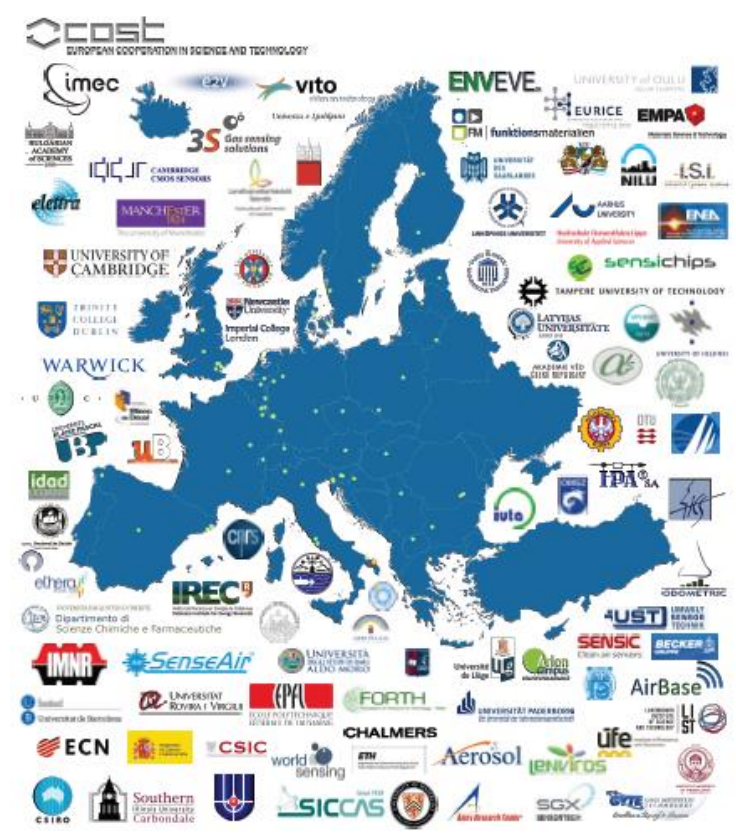

Fig. 5. Partnership of the COST Action TD1105. 


\section{S\&T Challenges}

The Action EuNetAir addresses significant challenges in science and technology in the field of air quality monitoring and environmental technologies:

- Nanomaterials for AQC sensors

- Low-cost Gas Sensors

- Low-power Sensor-Systems

- Wireless Technology (Environmental Sensors Network)

- Air Quality Modelling

- Environmental Measurements

- Standards and Protocols

\section{Innovation Highlights}

The Working Program includes multidisciplinary research at integrated approach and transdomain multi-scale level:

- Nanomaterials for low-cost AQC sensors

- Improved gas sensor systems and lowpower sensing microdevices

- Wireless sensor networks and distributed intelligence

- Air-quality modelling and chemical weather forecasting

- New protocols, standards and methods for AQC sensors

- Harmonisation of environmental measurements

- Guidelines for AQC systems and transducers

- Environmental sustainability and energy efficiency

\section{Action Deliverables}

The COST Action TD1105 is committed to deliver outcome during the four-year period (2012-2016):

- Workshops on sensor materials and nanotechnologies, sensor-systems for AQC, environmental measurements, air-pollution modelling, chemical weather forecasting, distributed computing, wireless sensor networks, protocols and pre-standardisation; organization of open conferences to improve knowledge transfer and dissemination

- Training Schools on sensor materials, technologies, processes, methods, modelling, forecasting, applications, environmental certification and validation, project management

- International ESRs exchange and Scientists Mobility (STSMs) between partners involved in Action and Non-COST partnership at incoming/outcoming level

- New collaborative research initiatives and research projects providing synergies between partners capabilities
- Participations in Conferences, Short Courses, Mutual Publications, Reports, White Papers, Position Papers, etc.

- Outreach activities

- Enforcement of the Gender Balance agenda

- Coordinated Dissemination of the networking activities towards Academia, Industry and General Public

\section{Expected Impact}

The COST Action TD1105 is expected to impact as follows as:

- European Leadership on AQC Science \& Technology

- Development of Green-Economy

- Support to Sustainable Development

- Support to Monitoring System of Clean Air for Europe

- Fostering Research \& Innovation on New Sensing Technologies for Environmental Monitoring

\section{Conclusions and Outlook}

The COST Action TD1105 EuNetAir is proposed to solve problems in the area of:

- Air Quality Control

- Environmental Sustainability

- Indoor/Outdoor Energy Efficiency

- Climate Change Monitoring

- Health Effects of Air-Pollution

The Action is working to elaborate a Strategic Report devoted to the Innovation on Environmental Sensor Technologies, expected to be published on the end of 2016 .

\section{Acknowledgements}

The EuNetAir Consortium thanks the COST Association for networking funding (COST Action TD1105) and the COST Officers for continuous support to manage this successful COST Action TD1105.

\section{References}

[1] Action Memorandum of Understanding (MoU): www.cost.eu/domains_actions/essem/Actions/TD 1105

[2] Action website: http://www.cost.eunetair.it

[3] M. Penza et al., COST Action TD1105: New Sensing Technologies for Environmental Sustainability in Smart Cities, IEEE Sensors 2014 Proceedings, Valencia, Spain, 2 - 5 November 2014. DOI: 978-1-4799-0162-3/14/\$31.00@2014 IEEE.

[4] M. Penza, COST Action TD1105: Overview of Sensor-Systems for Air Quality Monitoring, Procedia Engineering 87 (2014) 1370-1377. 